\title{
Methicillin-Resistant Staphylococcus aureus Bloodstream Infections and Injection Drug Use, Tennessee, USA, 2015-2017
}

\author{
Meghana P. Parikh, Rany Octaria, Marion A. Kainer
}

\begin{abstract}
Medscape ACTIVITY
In support of improving patient care, this activity has been planned and implemented by Medscape, LLC and Emerging Infectious Diseases. Medscape, LLC is jointly accredited by the Accreditation Council for Continuing Medical Education (ACCME), the Accreditation Council for Pharmacy Education (ACPE), and the American Nurses Credentialing Center (ANCC), to provide continuing education for the healthcare team.

Medscape, LLC designates this Journal-based CME activity for a maximum of 1.00 AMA PRA Category 1 Credit(s) ${ }^{\mathrm{TM}}$. Physicians should claim only the credit commensurate with the extent of their participation in the activity. Successful completion of this CME activity, which includes participation in the evaluation component, enables the participant to earn up to $1.0 \mathrm{MOC}$ points in the American Board of Internal Medicine's (ABIM) Maintenance of Certification (MOC) program. Participants will earn MOC points equivalent to the amount of CME credits claimed for the activity. It is the CME activity provider's responsibility to submit participant completion information to ACCME for the purpose of granting ABIM MOC credit.

All other clinicians completing this activity will be issued a certificate of participation. To participate in this journal CME activity: (1) review the learning objectives and author disclosures; (2) study the education content; (3) take the post-test with a $75 \%$ minimum passing score and complete the evaluation at http://www.medscape.org/journal/eid; and (4) view/print certificate. For CME questions, see page XXX.
\end{abstract}

Release date: February 12, 2020; Expiration date: February 12, 2021

\section{Learning Objectives}

Upon completion of this activity, participants will be able to:

- Describe the prevalence of life-threatening IDU-related MRSA BSI in Tennessee in 2015-2017, according to an analysis of data from the NHSN and the Tennessee Hospital Discharge Data System

- Determine the demographics and clinical characteristics of IDU-related MRSA BSI in Tennessee in 2015-2017, according to an analysis of data from the NHSN and the Tennessee Hospital Discharge Data System

- Identify clinical and public health implications of the association of IDU practices with life-threatening MRSA BSI in Tennessee, according to an analysis of data from the NHSN and the Tennessee Hospital Discharge Data System

\section{CME Editor}

Deborah Wenger, MBA, Copyeditor, Emerging Infectious Diseases. Disclosure: Deborah Wenger, MBA, has disclosed no relevant financial relationships.

\section{CME Author}

Laurie Barclay, MD, freelance writer and reviewer, Medscape, LLC. Disclosure: Laurie Barclay, MD, has disclosed no relevant financial relationships.

\section{Authors}

Disclosures: Meghana Pendurthi Parikh, VMD, MPH; and Rany Octaria, MD, MPH, have disclosed no relevant financial relationships. Marion Kainer, MBBS, MPH, has disclosed the following relevant financial relationships: served as an advisor or consultant for Infectious Disease Consulting Corporation; Pfizer Inc.

Author affiliations: Tennessee Department of Health, Nashville, Tennessee, USA (M.P. Parikh, R. Octaria, M.A. Kainer); Vanderbilt University, Nashville (R. Octaria) 
Recently, Tennessee, USA, has seen an increase in the use of commonly injected drugs, such as heroin and fentanyl. Injection drug use (IDU) practices can lead to life-threatening methicillin-resistant Staphylococcus aureus (MRSA) bloodstream infections (BSIs) and other serious diseases. We matched MRSA BSIs identified through the National Healthcare Safety Network to the Tennessee Hospital Discharge Data System to characterize the prevalence, demographics, and clinical characteristics associated with IDU in this disease population. Of the 7,646 MRSA BSIs identified during 2015-2017, we found that $1,839(24.1 \%)$ were IDU-related. IDUrelated BSIs increased by $118.9 \%$; the greatest rise occurred among emergency department-onset infections (197.4\%). IDU was more often associated with white, female, 18-49-year-old, and uninsured persons $(p<0.001)$. We found $\geq 1$ additional IDU-related diagnoses in $84.2 \%$ of IDU-related BSIs. Targeted harm reduction strategies for persons at high risk of IDU are necessary to reduce MRSA BSIs in acute care settings.

$\mathrm{M}$ ethicillin-resistant Staphylococcus aureus (MRSA) continues to be a prominent healthcare-associated pathogen causing illness and death $(1,2)$. As a result of the widespread implementation of infection control practices in acute-care hospitals, nationwide decreases in hospital-onset MRSA (HO MRSA) bloodstream infections (BSIs) were seen during 2005-2012. However, recent data show that since then there has been no change in the incidence of HO MRSA BSIs (3). Surveillance data on MRSA BSIs from acute care hospitals in Tennessee show similar patterns in decline and stabilization of HO BSIs; however, state trends in community-onset (CO) BSIs vary greatly from reported national patterns. Nationwide estimates suggest that incidence of CO MRSA BSIs has remained stable during 2005-2016 (3), whereas Tennessee's statewide surveillance showed a $37.2 \%$ increase in CO MRSA BSI events during 2011-2016 (4).

CO MRSA BSIs, classified as having a positive blood sample collected on or before day 3 of hospitalization or during an emergency department (ED) visit (5), are often associated with previous healthcare procedures and hospitalizations $(6,7)$. Additional risk factors for CO MRSA among previously healthy persons include, but are not limited to, close contact with colonized or infected persons (8), shared equipment that is not cleaned between users (9), and skin trauma $(10,11)$. We postulate that Tennessee's unique epidemiology of CO MRSA BSIs might be reflective of geographic differences in injection drug use (IDU) practices associated with the opioid epidemic. These patients might have clinical manifestations and risk factors that vary from those identified in previous literature. In the 2000s, opioid use was largely associated with abuse of prescription opioids, but during the past decade, the rise in opioid use and overdose deaths has been attributed to an increase in commonly injected drugs such as heroin and fentanyl (12-14). In Tennessee, although prescription opioids are still responsible for the greatest number of opioid deaths, overdose deaths associated with synthetic opioids increased by $666 \%$ and overdose deaths associated with heroin increased by 522\% during 2012-2017 (15).

IDU has long been identified as a risk factor for invasive MRSA infections, including skin and soft tissue infections (16-18), osteomyelitis and septic arthritis (19-21), bacteremia $(17,22)$, and endocarditis $(18,23,24)$. Data from 6 sites of the Centers for Disease Control and Prevention's Emerging Infections Program (Division of Preparedness and Emerging Infections, National Center for Emerging and Zoonotic Infectious Diseases) have shown that persons who inject drugs are $>16$ times more likely to develop invasive MRSA infections than persons who do not inject drugs and that the proportion of invasive MRSA associated with IDU has risen from 4.1\% in 2012 to $9.2 \%$ in $2016(10)$.

We sought to describe the prevalence of IDUrelated MRSA BSI cases in acute-care hospitals across Tennessee. In addition, we examined the demographic and clinical characteristics of IDU-related and non-IDU-related cases. With these data, we aim to inform targeted efforts to improve clinical response to high-risk MRSA BSI patients in both outpatient and inpatient settings. Furthermore, increased knowledge of the indirect impacts of the opioid epidemic is imperative for the development of policy-based prevention initiatives.

\section{Methods}

\section{Data Sources}

We identified MRSA BSIs using the National Healthcare Safety Network (NHSN), a nationwide reporting system through which acute care hospitals in Tennessee track laboratory-identified MRSA BSIs from inpatient (IP) units and EDs (5). NHSN includes details on specimen collection and facility characteristics, in addition to limited patient identifiers, such as sex, date of birth, and name (optional to report).

The Tennessee Hospital Discharge Data System (HDDS) was used to further characterize demographics and clinical characteristics of MRSA BSIs identified in NHSN. HDDS captures administrative data on patient demographics, diagnoses, and procedures 
performed during all IP hospitalizations and ED encounters occurring in Tennessee hospitals during January 2014-June 2018 (25). In that time frame, all hospital visits for MRSA BSI patients were identified by matching records on patient names, dates of birth, or medical record numbers when other identifiers were unavailable. Beginning in January 2016, all HDDS diagnosis codes were documented using the International Classification of Diseases (ICD), 10th Revision, Clinical Modification (ICD-10-CM). Prior to that, codes from ICD-10-CM or the ICD's Ninth Revision, Clinical Modification (ICD-9-CM) were allowed in HDDS.

Our study cohort included MRSA BSIs from patients $\geq 13$ years of age with onset of infection during January 2015-December 2017 and with $>1$ IP or ED visit to any Tennessee hospital during July 2014-June 2018, as identified in HDDS. HDDS observations were excluded if full patient names or dates of birth were missing.

\section{Variables}

We classified a case of MRSA BSI as IDU-related if any HDDS visit in the 6 months before or after blood specimen collection contained a diagnosis code for drug use (primary or secondary). The list of ICD codes used included diagnoses for dependence, abuse, poisoning, or accidental death caused by commonly injected illicit drugs (e.g., cocaine, opioids, methamphetamine) (Appendix Table 1, https://wwwnc.cdc.gov/EID/article/26/3/19-1408-App1.pdf). These codes have been used in peer-reviewed literature to estimate IDU associated with hospitalizations for other infectious diseases, such as infective endocarditis $(24,25,27)$.

In accordance with NHSN guidelines, we classified a BSI event as CO if the culture was obtained on or before hospital day 3 and as $\mathrm{HO}$ if obtained on hospital day 4 or later, with the admission date being day 1 (5). We further classified CO infections as either CO-ED or CO-IP on the basis of the patient's location at the time of culture collection. We classified sameday cultures collected in both ED and IP locations as a single CO-ED event.

We also evaluated cases for the presence of other IDU-related diagnoses in the 6 months before or after blood collection, including hospitalization for MRSA BSI. Thus, these cases could have occurred as a part of the same or different disease process as the BSI event. IDU-related diagnosis codes included endocarditis, acute or chronic hepatitis C, osteomyelitis or septic arthritis, and skin and soft tissue infections (Appendix Table 2).

\section{Statistical Analysis}

We evaluated differences in baseline characteristics between IDU-related and non-IDU-related BSIs using a $\chi^{2}$ or Fisher exact test for categorical variables and 2-sample $t$-test for continuous variables. We further analyzed IDU-related MRSA BSI events by onset group, using a $\chi^{2}$ or Fisher exact test for categorical variables and 1-way analysis of variance for continuous variables. We performed database linkages and statistical analyses using SAS 9.4 (SAS Institute Inc., https://www.sas.com). We defined statistical significance as $\mathrm{p}<0.05$. This study was approved by the Tennessee Department of Health (TDH) Institutional Review Board (project no. 1148777-1).

\section{Results}

After excluding patients $<13$ years of age at the time of culture, we identified 8,251 NHSN MRSA BSI cases from 7,076 patients during 2015-2017. Of those patients, 6,548 (92.5\%) were located within HDDS. In total, the matched patients represented 7,646 MRSA BSI cases included in the study cohort. We identified only 1 BSI case per person in $87.5 \%$ of patients; the maximum number of BSI events per person over the study timeframe was 8. Tennessee state residents had $89.7 \%$ of BSIs.

MRSA BSI cases increased $17.7 \%$ over the study period, from 2,333 cases in 2015 to 2,746 in 2017 (Figure). Most cases (57\%) were CO-ED. During 2015-2017, CO-ED BSIs increased by $51.8 \%$, as compared with decreases in CO-IP (-17.9\%) and HO (-11.4\%) BSIs.

IDU-related cases represented $24.1 \%$ of the study cohort; the prevalence of these cases increased from $16.1 \%$ in 2015 to $29.9 \%$ in 2017 (Table 1). The proportion of IDU-related cases was highest among the CO-ED group $(26.5 \%)$ compared with other onset groups $(p<0.001)$. Age was associated with IDU status $(p<0.001)$; the median age of patients with

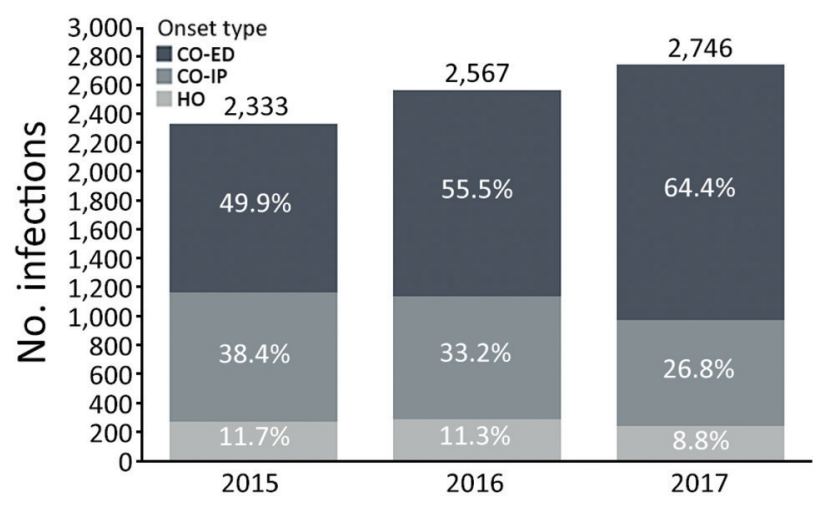

Figure. Annual cases of methicillin-resistant Staphylococcus aureus bloodstream infections in hospitals, stratified by onset type, Tennessee, USA, 2015-2017. CO, community onset; ED, emergency department; HO, hospital onset; IP, inpatient. 
Table 1. Demographic and clinical characteristics of patients with methicillin-resistant Staphylococcus aureus bloodstream infections, by IDU status, Tennessee, USA, 2015-2017*

\begin{tabular}{|c|c|c|c|c|}
\hline \multirow[b]{2}{*}{ Characteristic } & \multicolumn{2}{|c|}{ IDU status } & \multirow[b]{2}{*}{$p$ value } & \multirow[b]{2}{*}{ Overall } \\
\hline & IDU & Non-IDU & & \\
\hline Total & $1,839(24.1)$ & 5,807 (75.9) & $<0.001$ & $7,646(100)$ \\
\hline Onset year & & & $<0.001$ & \\
\hline 2015 & $375(16.1)$ & $1,958(83.9)$ & & $2,333(30.5)$ \\
\hline 2016 & $643(25.0)$ & $1,924(75.0)$ & & $2,567(33.6)$ \\
\hline 2017 & $821(29.9)$ & $1,925(70.1)$ & & $2,746(35.9)$ \\
\hline Onset type & & & $<0.001$ & \\
\hline CO-ED & $1,156(62.9)$ & $3,202(55.1)$ & & $4,358(57.0)$ \\
\hline CO-IP & $572(31.1)$ & $1,911(32.9)$ & & $2,483(32.5)$ \\
\hline $\mathrm{HO}$ & $111(6.0)$ & $694(12.0)$ & & $805(10.5)$ \\
\hline Age range, $y$ & & & $<0.001$ & \\
\hline $13-17$ & $1(0.1)$ & $24(0.4)$ & & $25(0.3)$ \\
\hline $18-34$ & $617(33.6)$ & $387(6.7)$ & & $1,004(13.1)$ \\
\hline $35-49$ & $665(36.2)$ & $912(15.7)$ & & $1,577(20.63)$ \\
\hline $50-64$ & $436(23.7)$ & $1,789(30.8)$ & & $2,225(29.1)$ \\
\hline$\geq 65$ & $120(6.5)$ & $2,695(46.4)$ & & $2,815(36.8)$ \\
\hline Median (range, Q1-Q3) & $40(31-51)$ & $63(51-74)$ & $<0.001$ & \\
\hline Sex & & & $<0.001$ & \\
\hline $\mathrm{M}$ & $910(49.5)$ & $3,435(59.2)$ & & $4,345(56.8)$ \\
\hline $\mathrm{F}$ & $929(50.5)$ & $2,372(40.8)$ & & $3,301(43.2)$ \\
\hline Race & & & $<0.001$ & \\
\hline White & $1,635(88.9)$ & $4,511(77.7)$ & & $6,146(80.4)$ \\
\hline Black & $168(9.1)$ & 1,193 (20.5) & & $1,361(17.8)$ \\
\hline Other & $15(0.8)$ & $56(1.0)$ & & $71(0.9)$ \\
\hline Unknown & 21 (1.1) & $47(0.8)$ & & $68(0.9)$ \\
\hline Ethnicity & & & 0.820 & \\
\hline Hispanic & $6(0.3)$ & $22(0.4)$ & & $28(0.4)$ \\
\hline Non-Hispanic & 1,768 (96.1) & $5,563(95.8)$ & & 7,331 (95.9) \\
\hline Unknown & $65(3.5)$ & $222(3.8)$ & & $287(3.8)$ \\
\hline Insurance & & & $<0.001$ & \\
\hline Commercial & $109(5.9)$ & $648(11.2)$ & & 757 (9.9) \\
\hline Medicaid & $570(31.0)$ & 655 (11.3) & & $1,225(16.0)$ \\
\hline Medicare & $452(24.6)$ & 3,688 (63.5) & & $4,140(54.2)$ \\
\hline Self-pay/uninsured & 612 (33.3) & $476(8.2)$ & & $1,088(14.2)$ \\
\hline Other/unknown & $96(5.2)$ & $340(5.9)$ & & $436(5.7)$ \\
\hline
\end{tabular}

*Values are no. (\%) except as indicated. CO, community onset; ED, emergency department; HO, hospital onset; IDU, injection drug use; IP, inpatient.

IDU-related BSIs was 40 years versus 63 years for patients with non-IDU-related BSI cases. Among IDUrelated cases, $69.7 \%$ of BSIs occurred in 18-49-yearolds, whereas the same age range made up only $22.4 \%$ of non-IDU-related BSIs. Gender was also correlated with IDU status $(p<0.001)$; men accounted for a smaller proportion of IDU-related BSIs (49.5\%) than nonIDU-related BSIs (59.2\%). Of all MRSA BSIs, $80.4 \%$ occurred in white patients and $17.8 \%$ in black patients. The proportion of white patients was higher among IDU-related cases than among non-IDU-related cases $(88.9 \%$ versus $77.7 \%$; $p<0.001)$. Usage of Medicare and commercial insurance was higher among non-IDUrelated BSIs (63.5\% vs. $11.2 \%$ for IDU-related BSIs), whereas Medicaid usage and self-pay/uninsured status were higher among IDU-related cases $(31.0 \%$ vs. $33.3 \%$ for non-IDU-related cases).

Among all patients with MRSA BSIs, 4,604 $(61.8 \%)$ had $\geq 1$ IDU-related diagnoses documented within 6 months before or after MRSA onset. Prevalence of IDU-related diagnoses was $84.2 \%$ among patients with IDU-related BSIs and $54.7 \%$ among those with non-IDU-related BSIs. The prevalence of endocarditis (40.4\%), hepatitis C infections (50.7\%), osteomyelitis/septic arthritis (28.1\%), and skin and soft tissue infections $(46.9 \%)$ were all significantly greater $(\mathrm{p}<0.001$ for all $)$ among IDU-related BSIs than among non-IDU-related BSIs (Table 2).

Among IDU-related cases stratified by onset type, $62.9 \%$ were CO-ED (Table 3 ). The proportion of

$\begin{aligned} & \text { Table 2. Prevalence of IDU-related diagnoses among patients with methicillin-resistant Staphylococcus aureus bloodstream infections, } \\
& \text { by IDU status, Tennessee, USA, 2015-2017* }\end{aligned}$
\begin{tabular}{lcccc}
\hline Diagnosis & IDU, no. (\%), $\mathrm{n}=1,839$ & Non-IDU, no. (\%), $\mathrm{n}=5,807$ & p value & Overall, no. (\%), $\mathrm{n}=7,646$ \\
\hline Endocarditis & $743(40.4)$ & $626(10.8)$ & $<0.001$ & $1,369(17.9)$ \\
Hepatitis C & $932(50.7)$ & $377(6.5)$ & $<0.001$ & $1,309(17.1)$ \\
Osteomyelitis/septic arthritis & $516(28.1)$ & $1,340(23.1)$ & $<0.001$ & $1,856(24.3)$ \\
Skin/soft tissue infection & $863(46.9)$ & $2,227(38.4)$ & $<0.001$ & $3,090(40.4)$ \\
\hline
\end{tabular}


Table 3. Selected characteristics of patients with injection drug use-related methicillin-resistant Staphylococcus aureus bloodstream infections, by onset type, Tennessee, USA, 2015-2017*

\begin{tabular}{|c|c|c|c|c|c|}
\hline \multirow[b]{2}{*}{ Characteristic } & \multicolumn{3}{|c|}{ MRSA onset } & \multirow[b]{2}{*}{$p$ value } & \multirow[b]{2}{*}{ Overall } \\
\hline & CO-ED & CO-IP & $\mathrm{HO}$ & & \\
\hline Total & $1,156(62.9)$ & $572(31.1)$ & $111(6.0)$ & $<0.001$ & $1,839(100)$ \\
\hline Onset year & & & & $<0.001$ & \\
\hline 2015 & $192(51.2)$ & $153(40.8)$ & $30(8.0)$ & & $375(20.4)$ \\
\hline 2016 & $393(61.1)$ & $211(32.8)$ & $39(6.1)$ & & $643(35.0)$ \\
\hline 2017 & $571(69.5)$ & $208(25.3)$ & $42(5.1)$ & & $821(44.6)$ \\
\hline Age range, $y$ & & & & $<0.001$ & \\
\hline $13-17$ & 0 & $1(0.2)$ & 0 & & $1(0.0)$ \\
\hline $18-34$ & $431(37.3)$ & $164(28.7)$ & $22(19.8)$ & & $617(33.6)$ \\
\hline $35-49$ & $405(35.0)$ & $226(39.5)$ & $34(30.6)$ & & $665(36.2)$ \\
\hline $50-64$ & $245(21.2)$ & $149(26.0)$ & $42(37.8)$ & & $436(23.7)$ \\
\hline$>65$ & $75(6.5)$ & $32(5.6)$ & $13(11.7)$ & & $120(6.5)$ \\
\hline Median (Q1-Q3) & $38(31-51)$ & $42(33-52)$ & $49(37-59)$ & $<0.001$ & \\
\hline Race & & & & $<0.001$ & \\
\hline White & $1,059(91.6)$ & $501(87.6)$ & $75(67.6)$ & & 1,635 (88.9) \\
\hline Black & $83(7.2)$ & $56(9.8)$ & $29(26.1)$ & & $168(9.1)$ \\
\hline Other & $6(0.5)$ & $7(1.2)$ & $2(1.8)$ & & $15(0.8)$ \\
\hline Unknown & $8(0.7)$ & $8(1.4)$ & $5(4.5)$ & & $21(1.1)$ \\
\hline Insurance & & & & 0.001 & \\
\hline Commercial & $69(6.0)$ & $32(5.6)$ & $8(7.2)$ & & $109(5.9)$ \\
\hline Medicaid & $347(30.0)$ & $192(33.6)$ & $31(27.9)$ & & 570 (31.0) \\
\hline Medicare & $256(22.1)$ & $154(26.9)$ & $42(37.8)$ & & $452(24.6)$ \\
\hline Self-pay/uninsured & $422(36.5)$ & $164(28.7)$ & $26(23.4)$ & & $612(33.3)$ \\
\hline Other/unknown & $62(5.4)$ & $30(5.2)$ & $4(3.6)$ & & $96(5.2)$ \\
\hline
\end{tabular}

CO-ED cases increased by $18.4 \%$ during 2015-2017, whereas the proportion of CO-IP cases among IDUrelated BSIs decreased by $15.5 \%$ and the proportion of HO cases among IDU-related BSIs decreased by $2.9 \%$. CO-ED IDU-related BSIs had the youngest patients, with a median age of 38 years $(\mathrm{p}<0.001)$. Onset type among IDU-related BSIs was associated with insurance status $(p=0.001)$; the greatest usage of Medicare $(37.8 \%)$ and commercial insurance $(7.2 \%)$ occurred among $\mathrm{HO}$ cases, whereas self-pay/uninsured status was highest among CO-ED cases (36.5\%). Medicaid was used most often among patients with CO-IP IDU-related BSIs (33.6\%).

\section{Discussion}

We found an alarming increase in the extent of all MRSA BSIs in Tennessee during 2015-2017. This rise is attributed largely to the increase in the number of CO-ED cases, as CO-IP and HO cases have steadily declined. Increasing IDU over the study timeframe, as well as the high prevalence of IDU among CO-ED BSIs, suggests an association between the drug use crisis and MRSA BSIs. These trends are consistent with reports of increasing use of commonly injected drugs in Tennessee based on the surveillance of overdose deaths $(14,15)$, which might provide an incomplete picture of current drug use practices. The use of hospital discharge billing data in our study enabled us to assess IDU among all patients entering the hospital system, including those who survived.
Using this methodology, we described common demographic characteristics of MRSA BSI patients and stratified them by IDU status. Consistent with previously reported demographics associated with IDU (12), we observed that IDU in our population was more common among patients who were 18-49 years of age, female, white, and uninsured. Furthermore, although still observed in the CO-IP and HO groups, IDU and those demographics were most strongly associated with ED-onset BSIs. Our findings demonstrate a shift in patient demographics typically associated with MRSA. Whereas previous studies have shown that invasive MRSA infections occur predominantly in men $>49$ years of age, with a larger proportion of patients being black (27), our study highlights an emerging at-risk population.

Currently, most public health MRSA BSI prevention and treatment strategies are targeted at $\mathrm{HO}$ infections $(3,28)$. The results of this study provide a compelling argument to enhance our MRSA BSI reduction efforts by devoting resources and creating policies targeting CO BSIs. First, this new knowledge can be used to heighten awareness in ED staff of potential IDU among patients with clinical signs consistent with MRSA BSIs. These patients have a high prevalence of other IDU-related diagnoses, including endocarditis and hepatitis C, which might affect clinical progression and, ultimately, patient outcomes. Identifying patients at risk for IDU-related MRSA BSIs enables prompt diagnosis, 
treatment, and increased emphasis on feasible follow-up care solutions.

A key difference between both CO groups in this study was the larger utilization of Medicaid among CO-IP IDU-related cases, compared with the higher rates of uninsurance among IDU-related CO-ED BSIs. This contrast has implications for follow-up care, because patients with IDU-related disease might be less likely to afford and pursue required follow-up treatment. In addition to the high potential for illness and death, these patients demonstrate higher rates of IDU-related infections and readmission (29), which are often also associated with uninsured status (19).

Our findings also raise a question about the role of ED and IP healthcare services in facilitating treatment for drug use and addiction. Despite evidence that interventions such as medication-assisted therapy and screening, brief intervention, and referral to treatment are both feasible and effective in acute care settings (30-32), pharmacotherapies and psychotherapies are heavily underused $(29,33)$. Implementing interventions for substance abuse in ED settings has a large potential impact on reducing CO MRSA BSIs and other devastating consequences of IDU.

Our findings are subject to some limitations. The events included in our analyses were laboratoryidentified cases from acute care hospitals sourced from Tennessee statewide surveillance data. In addition, only patients who were able to be matched to HDDS were included in the analyses; the match rate of $92.5 \%$ indicates a possible underrepresentation of the true burden of disease. We also recognize that because ICD codes do not differentiate between routes of administration for drug use, we might be overestimating the prevalence of IDU compared with overall substance abuse. Similarly, because of stigmas surrounding substance abuse, ICD codes documenting the practice might provide an underestimation of true prevalence. Although we were unable to access medical records to validate our approach, this series of diagnostic codes has previously been used to identify IDU related to infections and hospitalizations $(23,24,26)$. In addition, given that hepatitis $C$ is strongly correlated with IDU $(34,35)$, the high prevalence of hepatitis C infections among IDU-related MRSA BSI cases in this study lends support to the validity of the diagnostic codes used. For these reasons, it is feasible that our findings are reflective not only of patterns of substance abuse, but also of IDU in Tennessee.

Our study is unique in its linkage of NHSN MRSA BSI surveillance to hospital discharge data for retrospective evaluation of IDU without conducting time-consuming chart reviews. The use of statewide laboratory-based surveillance data provides the additional benefit of a more reliable, complete picture of MRSA BSIs across Tennessee. Previous studies relied on extrapolating data from smaller jurisdictions to estimate the burden of infection and describe patient characteristics, leaving the potential for inaccurate estimation and interpretation of state trends (10, 27). Our technique is advantageous for state public health agencies seeking to investigate the evolving clinical and demographic risk factors associated with reportable diseases. Despite reported national trends of unchanged CO MRSA BSIs (3), with the widespread nature of the opioid epidemic, we suspect that other jurisdictions, especially those with similar population characteristics as Tennessee, might see similar trends of rising CO MRSA BSIs associated with IDU. Replicating this study elsewhere would be valuable to identify any local variations in risk factors.

In summary, Tennessee is undergoing a major change in the epidemiology of MRSA BSIs, having a growing population of young, white, uninsured, female patients with CO BSIs as a consequence of IDU. Our findings can be used to inform public health policies and clinical practice, particularly in the ED setting, to introduce prevention and harm reduction strategies to reduce the widespread impacts of this deadly disease within our communities.

\section{Acknowledgments}

We thank the acute care hospitals in Tennessee for performing surveillance and reporting MRSA BSI data to NHSN.

Development of this manuscript and contents were supported through the Centers for Disease Control and Prevention (CDC) Epidemiology and Laboratory Capacity (grant no. 6NU50CK000386) and the CDC Emerging Infections Program (grant no. 6NU50CK000491).

\section{About the Author}

Dr. Parikh is an epidemiologist at the Tennessee Department of Health's Healthcare Associated Infections and Antimicrobial Resistance Program in Nashville, Tennessee, USA. Her primary research interests include surveillance of infectious diseases.

\section{References}

1. van Hal SJ, Jensen SO, Vaska VL, Espedido BA, Paterson DL, Gosbell IB. Predictors of mortality in Staphylococcus aureus bacteremia. Clin Microbiol Rev. 2012;25:362-86. https://doi.org/10.1128/CMR.05022-11

2. Sharma A, Rogers C, Rimland D, Stafford C, Satola S, Crispell E, et al. Post-discharge mortality in patients 
hospitalized with MRSA infection and/or colonization. Epidemiol Infect. 2013;141:1187-98. https:/ / doi.org/ $10.1017 /$ S0950268812001963

3. Kourtis AP, Hatfield K, Baggs J, Mu Y, See I, Epson E, et al.; Emerging Infections Program MRSA Author Group. Vital signs: epidemiology and recent trends in methicillinresistant and in methicillin-susceptible Staphylococcus aureus bloodstream infections - United States. MMWR Morb Mortal Wkly Rep. 2019;68:214-9. https://doi.org/10.15585/ mmwr.mm6809e1

4. Centers for Disease Control and Prevention. CDC Vital Signs town hall on staph infections can kill: prevention at the front lines; 2019 [cited 2019 Sep 10]. https:/ / www.cdc.gov/ publichealthgateway/townhall/2019/downloads/ 3-mar-presentation.pdf

5. National Center for Emerging and Zoonotic Infectious Diseases. Multidrug-resistant organism and Clostridioides difficile infection (MDRO/CDI) module. 2019 [cited 2019 Aug 6]. https://www.cdc.gov/nhsn/pdfs/ pscmanual/12pscmdro_cdadcurrent.pdf

6. Huang SS, Platt R. Risk of methicillin-resistant Staphylococcus aureus infection after previous infection or colonization. Clin Infect Dis. 2003;36:281-5. https:/ / doi.org/ 10.1086/345955

7. Huang SS, Hinrichsen VL, Datta R, Spurchise L, Miroshnik I, Nelson K, et al. Methicillin-resistant Staphylococcus aureus infection and hospitalization in highrisk patients in the year following detection. PLoS One. 2011; 6:e24340. https:/ / doi.org/10.1371/journal.pone.0024340

8. Campbell KM, Vaughn AF, Russell KL, Smith B, Jimenez DL, Barrozo CP, et al. Risk factors for communityassociated methicillin-resistant Staphylococcus aureus infections in an outbreak of disease among military trainees in San Diego, California, in 2002. J Clin Microbiol. 2004;42:4050-3. https:/ / doi.org/10.1128/JCM.42.9.40504053.2004

9. Centers for Disease Control and Prevention. Methicillinresistant Staphylococcus aureus infections among competitive sports participants - Colorado, Indiana, Pennsylvania, and Los Angeles County, 2000-2003. MMWR Morb Mortal Wkly Rep. 2003;52:793-5.

10. Jackson KA, Bohm MK, Brooks JT, Asher A, Nadle J, Bamberg WM, et al. Invasive methicillin-resistant Staphylococcus aureus infections among persons who inject drugs - six sites, 2005-2016. MMWR Morb Mortal Wkly Rep. 2018;67:625-8. https://doi.org/10.15585/mmwr.mm6722a2

11. Centers for Disease Control and Prevention (CDC). Methicillin-resistant Staphylococcus aureus skin infections among tattoo recipients - Ohio, Kentucky, and Vermont, 2004-2005. MMWR Morb Mortal Wkly Rep. 2006;55:677-9.

12. Cicero TJ, Ellis MS, Surratt HL, Kurtz SP. The changing face of heroin use in the United States: a retrospective analysis of the past 50 years. JAMA Psychiatry. 2014;71:821-6. https://doi.org/10.1001/jamapsychiatry.2014.366

13. Rudd RA, Seth P, David F, Scholl L. Increases in drug and opioid-involved overdose deaths - United States, 2010-2015. MMWR Morb Mortal Wkly Rep. 2016;65:1445-52. https:/ / doi.org/10.15585/mmwr.mm655051e1

14. Cicero TJ, Ellis MS, Harney J. Shifting patterns of prescription opioid and heroin abuse in the United States. N Engl J Med. 2015;373:1789-90. https:/ / doi.org/10.1056/ NEJMc1505541

15. National Institute on Drug Abuse. Tennessee opioid summary: opioid-related overdose deaths. 2018 [cited 2019 Aug 25]. https:/ / www.drugabuse.gov/drugs-abuse/opioids/ opioid-summaries-by-state/tennessee-opioid-summary.
16. Ebright JR, Pieper B. Skin and soft tissue infections in injection drug users. Infect Dis Clin North Am. 2002;16:697712. https:// doi.org/10.1016/S0891-5520(02)00017-X

17. Bassetti S, Battegay M. Staphylococcus aureus infections in injection drug users: risk factors and prevention strategies. Infection. 2004;32:163-9. https:// doi.org/10.1007/ s15010-004-3106-0

18. Gordon RJ, Lowy FD. Bacterial infections in drug users. N Engl J Med. 2005;353:1945-54. https://doi.org/10.1056/ NEJMra042823

19. Ronan MV, Herzig SJ. Hospitalizations related to opioid abuse/dependence and associated serious infections increased sharply, 2002-12. Health Aff (Millwood). 2016;35:832-7. https:// doi.org/10.1377/hlthaff.2015.1424

20. Chandrasekar PH, Narula AP. Bone and joint infections in intravenous drug abusers. Rev Infect Dis. 1986;8:904-11. https://doi.org/10.1093/clinids/8.6.904

21. Allison DC, Holtom PD, Patzakis MJ, Zalavras CG. Microbiology of bone and joint infections in injecting drug abusers. Clin Orthop Relat Res. 2010;468:2107-12. https://doi.org/10.1007/s11999-010-1271-2

22. Chua T, Moore CL, Perri MB, Donabedian SM, Masch W, Vager D, et al. Molecular epidemiology of methicillinresistant Staphylococcus aureus bloodstream isolates in urban Detroit. J Clin Microbiol. 2008;46:2345-52. https:/ / doi.org/ 10.1128/JCM.00154-08

23. Cooper HLF, Brady JE, Ciccarone D, Tempalski B, Gostnell K, Friedman SR. Nationwide increase in the number of hospitalizations for illicit injection drug use-related infective endocarditis. Clin Infect Dis. 2007;45:1200-3. https:// doi.org/10.1086/522176

24. Wurcel AG, Anderson JE, Chui KKH, Skinner S, Knox TA, Snydman DR, et al. Increasing infectious endocarditis admissions among young people who inject drugs. Open Forum Infect Dis. 2016;3:ofw157. https://doi.org/10.1093/ ofid/ofw157

25. Tennessee Department of Health. Hospital discharge data system. August 2018 [cited 2019 Aug 25]. https:/ / www. tn.gov/health/health-program-areas/statistics/ special-reports/hdds.html

26. Miller AC, Polgreen PM. Many opportunities to record, diagnose, or treat injection drug-related infections are missed: a population-based cohort study of inpatient and emergency department settings. Clin Infect Dis. 2019;68:1166-75. https:// doi.org/10.1093/cid/ciy632

27. Dantes R, Mu Y, Belflower R, Aragon D, Dumyati G, Harrison LH, et al.; Emerging Infections Program-Active Bacterial Core Surveillance MRSA Surveillance Investigators. National burden of invasive methicillin-resistant Staphylococcus aureus infections, United States, 2011. JAMA Intern Med. 2013;173:1970-8.

28. Centers for Disease Control and Prevention. What CDC is doing to combat MRSA. 2019 [cited 2019 Nov 25]. https:/ / www.cdc.gov/mrsa/tracking

29. Rosenthal ES, Karchmer AW, Theisen-Toupal J, Castillo RA, Rowley CF. Suboptimal addiction interventions for patients hospitalized with injection drug use-associated infective endocarditis. Am J Med. 2016;129:481-5. https:/ / doi.org/ 10.1016/j.amjmed.2015.09.024

30. Hawk KF, Vaca FE, D'Onofrio G. Reducing fatal opioid overdose: prevention, treatment and harm reduction strategies. Yale J Biol Med. 2015;88:235-45.

31. Madras BK, Compton WM, Avula D, Stegbauer T, Stein JB, Clark HW. Screening, brief interventions, referral to treatment (SBIRT) for illicit drug and alcohol use at multiple healthcare sites: comparison at intake and 6 
months later. Drug Alcohol Depend. 2009;99:280-95. https://doi.org/10.1016/j.drugalcdep.2008.08.003

32. Trowbridge P, Weinstein ZM, Kerensky T, Roy P, Regan D, Samet JH, et al. Addiction consultation services - linking hospitalized patients to outpatient addiction treatment. J Subst Abuse Treat. 2017;79:1-5. https:/ / doi.org/10.1016/j.jsat.2017.05.007

33. Knudsen HK, Abraham AJ, Roman PM. Adoption and implementation of medications in addiction treatment programs. J Addict Med. 2011;5:21-7. https:/ / doi.org/ 10.1097/ADM.0b013e3181d41ddb

34. Nelson PK, Mathers BM, Cowie B, Hagan H, Des Jarlais D, Horyniak D, et al. Global epidemiology of hepatitis B and hepatitis $\mathrm{C}$ in people who inject drugs: results of systematic reviews. Lancet. 2011;378:571-83. https:// doi.org/10.1016/ S0140-6736(11)61097-0
35. Zibbell JE, Iqbal K, Patel RC, Suryaprasad A, Sanders KJ, Moore-Moravian L, et al.; Centers for Disease Control and Prevention. Increases in hepatitis $C$ virus infection related to injection drug use among persons aged $\leq 30$ years - Kentucky, Tennessee, Virginia, and West Virginia, 2006-2012. MMWR Morb Mortal Wkly Rep. 2015;64:453-8.

Address for correspondence: Meghana P. Parikh, Tennessee Department of Health, Healthcare Associated Infections and Antimicrobial Resistance Program, 710 James Robertson Pkwy, Andrew Johnson Tower 3.421C, Nashville, TN 37203, USA; email: Meghana.Parikh@tn.gov

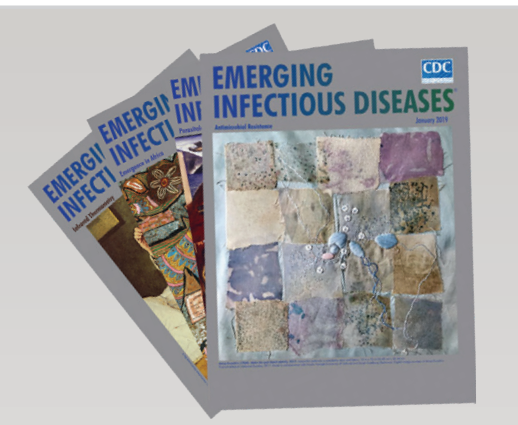

EMERGING INFECTIOUS DISEASES

January 2019

\section{Antimicrobial Resistance}

- Complexity of the Basic Reproduction Number $\left(R_{0}\right)$

- Aeromedical Transfer of Patients with Viral Hemorrhagic Fever

- Clinical and Radiologic Characteristics of Human Metapneumovirus Infections in Adults, South Korea

- Enterovirus A71 Infection and Neurologic Disease, Madrid, Spain, 2016

- Epidemiology of Imported Infectious Diseases, China, 2005-2016

- Risk Factors for Elizabethkingia Acquisition and Clinical

Characteristics of Patients, South Korea

- Effects of Antibiotic Cycling Policy on Incidence of HealthcareAssociated MRSA and Clostridioides difficile Infection in Secondary Healthcare Settings

- Association of Increased Receptor-Binding Avidity of Influenza A(H9N2) Viruses with Escape from Antibody-Based Immunity and Enhanced Zoonotic Potential

- Variable Protease-Sensitive Prionopathy Transmission to Bank Voles

- Zoonotic Source Attribution of Salmonella enterica Serotype Typhimurium Using Genomic Surveillance Data, United States

- Multiple Introductions of Domestic Cat Feline Leukemia Virus in Endangered Florida Panthers

- Prescription of Antibacterial Drugs for HIV-Exposed, Uninfected Infants, Malawi, 2004-2010

- Influenza H5/H7 Virus Vaccination in Poultry and Reduction of Zoonotic Infections, Guangdong Province, China, 2017-18

- Higher Viral Load of Emerging Norovirus GII.P16-GII.2 than Pandemic GII.4 and Epidemic GII.17, Hong Kong, China

- Autochthonous Transmission of Coccidioides in Animals, Washington, USA
- Meat and Fish as Sources of Extended-Spectrum $\beta$-LactamaseProducing Escherichia coli, Cambodia

- Oral Transmission of Trypanosoma cruzi, Brazilian Amazon

- Avian Influenza A(H9N2) Virus in Poultry Worker, Pakistan, 2015

- Puumala Hantavirus Genotypes in Humans, France, 2012-2016

- New Multidrug-Resistant Salmonella enterica Serovar Anatum Clone, Taiwan, 2015-2017

- Seroepidemiology of Parechovirus A3 Neutralizing Antibodies, Australia, the Netherlands, and United States

- Identification of Lonepinella sp. in Koala Bite Wound Infections, Queensland, Australia

- Surgical Site Infections Caused by Highly Virulent MethicillinResistant Staphylococcus aureus Sequence Type 398, China

- Canine Influenza Virus A(H3N2) Clade with Antigenic Variation, China, 2016-2017

- Isolation and Full-Genome Characterization of Nipah Viruses from Bats, Bangladesh

- Burdens of Invasive Methicillin-Susceptible and Methicillin-Resistant Staphylococcus aureus Disease, Minnesota, USA

- Dengue Virus IgM Serotyping by ELISA with Recombinant Mutant Envelope Proteins

- Orogenital Transmission of Neisseria meningitidis Causing Acute Urethritis in Men Who Have Sex with Men

- Trends in Azole Resistance in Aspergillus fumigatus, the Netherlands, 1994-2016

- Using the Health Belief Model to Analyze Instagram Posts about Zika for Public Health Communications 